\title{
On Finding the Smallest Generalized Eigenpair Using Markov Chain Monte Carlo Algorithm
}

\author{
Farshid Mehrdoust \\ Department of Applied Mathematics, Faculty of Mathematical Sciences, University of Guilan, Rasht, Iran \\ Email: fmehrdoust@guilan.ac.ir
}

Received January 24, 2011; revised May 7, 2012; accepted May 15, 2012

\begin{abstract}
This paper proposes a new technique based on inverse Markov chain Monte Carlo algorithm for finding the smallest generalized eigenpair of the large scale matrices. Some numerical examples show that the proposed method is efficient.
\end{abstract}

Keywords: Monte Carlo Method; Markov Chain; Generalized Eigenpair; Inverse Monte Carlo Algorithm

\section{Introduction}

Monte Carlo and quasi Monte Carlo methods comprise that branch of experimental mathematics which is concerned with experiments on random numbers. In the last decade they have found extensive use in the fields of operational research and nuclear physics, where there are a variety of problems beyond the available resources of theoretical mathematics [1,2]. The problem of calculating the largest or smallest generalized eigenvalue problem is one of the most important problems in science and engineering. This problem arises naturally in many applications. Mathematically it is a generalization of the symmetric eigenvalue problem and it can be reduced to an equivalent symmetric eigenvalue problem. Recently, evaluating the smallest and largest eigenpair of large scale matrices using Monte Carlo and quasi Monte Carlo methods has been studied [3-5].

Let $A, B \in \mathbb{R}^{n \times n}$ are real symmetric matrices and the matrix $B$ is a positive definite matrix. Consider the problem of evaluating the smallest eigenpair of the pencil ( $A$, $B)$ i.e. the values for which

$$
A x=\lambda B x
$$

A generalized eigenvalue problem (1) is said to be symmetric positive definite (S/PD) if $A$ is symmetric and $B$ is positive definite.

\section{Markov Chain Monte Carlo Algorithm}

Suppose that the matrix $A \in \mathbb{R}^{n \times n}$ and two vectors $f, g \in \mathbb{R}^{n}$ are given. Consider the following Markov chain $T_{i}$ with length $i$

$$
T_{i}: k_{0} \rightarrow k_{1} \rightarrow \cdots \rightarrow k_{i}
$$

where for $k_{j} \in\{1,2, \cdots, n\}, j=1,2, \cdots, i$. The statistical nature of constructing the chain (2) follow as

$$
p\left(k_{0}=\alpha\right)=p_{\alpha}, p\left(k_{j}=\alpha \mid k_{j-1}=\alpha\right)=p_{\alpha \beta}
$$

where $p_{\alpha}$ and $p_{\alpha \beta}$ show the probability of starting chain at $\alpha$ and transition probability from state $\alpha$ to $\beta$, respectively. In other words, we have

$$
\sum_{\alpha=1}^{n} p_{\alpha}=1, \sum_{\beta=1}^{n} p_{\alpha \beta}=1, \alpha=1,2, \cdots, n .
$$

Now, define the following random variable

$$
\Theta(h)=\frac{h_{k_{0}}}{p_{k_{0}}} \sum_{j=0}^{\infty} W_{j} f_{k_{j}}
$$

where $W_{j}=W_{j-1} \frac{a_{k_{j-1} k_{j}}}{p_{k_{j-1} k_{j}}}, j=1,2, \cdots, i$ and $W_{0}=1$.

Theorem 1. Consider the linear system $A x=b$. Let us the nonsingular matrix $M \in \mathbb{R}^{n \times n}$, such that $M A=I-L \in \mathbb{R}^{n \times n}$, then the system can be presented in the following form

$$
x=L x+f
$$

where $f=M b$. Then under condition $\max _{1 \leq i \leq n} \sum_{j=1}^{n}\left|l_{i j}\right|<1$, the random variable $\Theta(h)$ is unbiased estimator, i.e.

$$
E[\Theta(h)]=\langle h, x\rangle \text {. }
$$

Proof [3].

Suppose that $x^{(i)}$ is the $i^{\text {th }}$ iterative solution of the following recursion relation with $x^{(0)}=f$. Now, we consider the random variable

$$
\Theta_{i}(h)=\frac{h_{k_{0}}}{p_{k_{0}}} \sum_{j=0}^{i} W_{j} f_{k_{j}}
$$


then $E\left[\Theta_{i}(h)\right]=\left\langle h, x^{(i+1)}\right\rangle$.

By simulating $N$ random paths with length $i$ :

$$
T_{i}^{(s)}: k_{0}^{(s)} \rightarrow k_{1}^{(s)} \rightarrow \cdots \rightarrow k_{i}^{(s)}
$$

we can write

$$
\Theta_{i}^{(s)}(h)=\frac{h_{k_{0}^{(s)}}}{p_{k_{0}^{(s)}}} \sum_{j=0}^{i} W_{j}^{(s)} f_{k_{j}^{(s)}}
$$

The Monte Carlo estimation can be evaluated by $\hat{\Theta}_{i}=\frac{1}{N} \sum_{s=1}^{N} \Theta_{i}^{(s)}(h)$, which is an approximation of $\left\langle h, x^{(i+1)}\right\rangle$.

Now, consider the following choice for the initial density vector and the transition probability matrix leads to an Almost Optimal Monte Carlo (AOMC) algorithm.

$$
p_{\alpha}=\frac{\left|h_{\alpha}\right|}{\sum_{\alpha=1}^{n}\left|h_{\alpha}\right|}, p_{\alpha \beta}=\frac{\left|a_{\alpha \beta}\right|}{\sum_{\beta=1}^{n}\left|a_{\alpha \beta}\right|}, \alpha=1,2, \cdots, n .
$$

Theorem 2. Using the above choice $p=\left(p_{\alpha}\right)_{\alpha=1}^{n}$ and $p=\left[p_{\alpha \beta}\right]_{\alpha, \beta=1}^{n}$ the variance of the unbiased estimator for obtaining the inverse matrix is minimized.

Proof [3].

\section{Inverse Monte Carlo Iterative (IMCI)}

Inverse Monte Carlo iterative algorithm can be applied when A is a nonsingular matrix. In this method, we calculate the following functional in each.

$$
\frac{\left\langle h_{j}, A f_{j}\right\rangle}{\left\langle h_{j}, B f_{j}\right\rangle}=\frac{\left\langle h_{j-1}, B f_{j}\right\rangle}{\left\langle h_{j}, B f_{j}\right\rangle}
$$

It is more efficient that we first evaluate the inverse matrix using the Monte Carlo algorithm [3,4]. The algorithm can be realized as follows

\section{The Proposed Algorithm}

1) Inputs $A \in \mathbb{R}^{n \times n}, \quad f_{0} \in \mathbb{R}^{n}$.

2) Starting from initial vector $f_{0}$.

3) For $j=1,2, \cdots$

4) Using global algorithm [4], Calculate the sequence of Monte Carlo iterations by solving the following System

Set

$$
A f_{j}=B f_{j-1}
$$

$$
\lambda_{j}=\frac{\left\langle h_{j}, A f_{j}\right\rangle}{\left\langle h_{j}, B f_{j}\right\rangle}=\frac{\left\langle h_{j-1}, B f_{j}\right\rangle}{\left\langle h_{j}, B f_{j}\right\rangle}
$$

5) Output: Smallest eigenvector $\lambda_{j}$ and the corresponding eigenvector $f_{j}$.
Table 1. Computing the smallest eigenpair for different matrices using MCMC algorithm.

\begin{tabular}{ccc}
\hline Matrix dimension & $\begin{array}{c}\text { Rel. error of } \\
\text { eigenvalue }\end{array}$ & $\begin{array}{c}\text { Rel. error of } \\
\text { eigenvector }\end{array}$ \\
\hline $64 \times 64$ & $1.26 \times 10 \mathrm{e}-7$ & $8.36 \times 10 \mathrm{e}-4$ \\
$128 \times 128$ & $1.18 \times 10 \mathrm{e}-7$ & $6.33 \times 10 \mathrm{e}-4$ \\
$256 \times 256$ & $6.48 \times 10 \mathrm{e}-8$ & $3.16 \times 10 \mathrm{e}-4$ \\
$512 \times 512$ & $7.91 \times 10 \mathrm{e}-8$ & $1.83 \times 10 \mathrm{e}-4$ \\
$1024 \times 1024$ & $7.04 \times 10 \mathrm{e}-8$ & $1.06 \times 10 \mathrm{e}-4$ \\
\hline
\end{tabular}

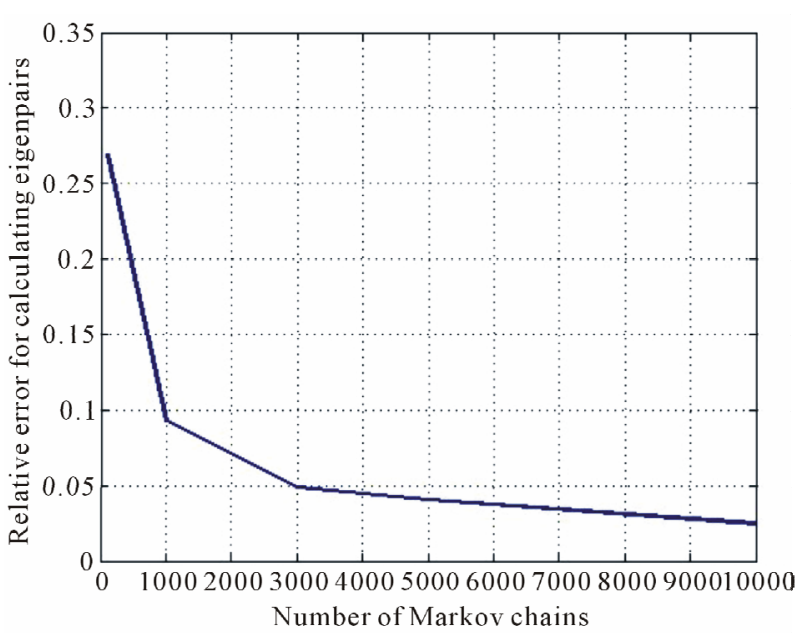

Figure 1. Relative error based on number of Markov chains.

6) End of Algorithm.

\section{Numerical Results}

In this section, the results listed in Table $\mathbf{1}$ are relative errors obtained by MCMC. In all of computations we used the MATLAB function eig (A, B) results in contrast to MCMC algorithm results.

\section{Conclusion}

A new numerical algorithm based on the inverse Markov chain Monte Carlo algorithm to calculate the smallest generalized eigenpair proposed in this paper. Also, we saw that by increasing the number of Markov chains the relative error is decreased (Figure 1).

\section{Acknowledgements}

The author is very grateful to two anonymous referees for giving very useful and valuable comments.

\section{REFERENCES}

[1] R. Y. Rubinstein, "Simulation and the Monte Carlo 
Method," John Wiley \& Sons, New York, 1981. doi:10.1002/9780470316511

[2] Y. Saad, "Numerical Methods for Large Eigenvalue Problems," Manchester University Press, Manchester, 1991.

[3] I. Dimov and A. Karaivanova, "Iterative Monte Carlo Algorithm for Linear Algebra Problem," Lecture Note in Computer Science, 1996.
[4] I. Dimov, "Monte Carlo Methods for Applied Scientists," World Scientific Pub., Singapore City, 2008.

[5] B. Fathi and F. Mehrdoust, "Partitioning Inverse Monte Carlo Iterative Algorithm for Finding the Three Smallest Eigenpairs of Generalized Eigenvalue Problem," Advances in Numerical Analysis, Vol. 2011, 2011, Article ID: 826376 . 